\title{
Evaluation of liposuction as a modality of minimal invasive treatment of gynecomastia
}

A.M.Abdelmofeed, M.A.Mansour, A.T.Salem and A.F.Ahmed

General surgery, Dept., Faculty of Medicine, Benha Univ., Benha, Egypt

E-mail: afarid508@yahoo.com

\begin{abstract}
Foreground: Gynecomastia occurs when the glandular tissue of the male breast grows abnormally, resulting in an abnormally big mass that extends concentrically from the base of the nipple. Objectives and goals Results of subcutaneous liposuction and glandular excision via an inframammary incision for the treatment of gynecomastia are the focus of this research. The department of general surgery at Benha University's college of medicine studied 20 teenage male patients aged 18-35 with gynecomastia ranging from grade I to early grade III. 50\% of men had gynecomastia on the right side, whereas $45 \%$ had just minor gynecomastia. Among those who took part in the study, just $20 \%$ had firm gynecomastia, while $55 \%$ had fair skin quality. Conclusion: Liposuction is usually the first step in surgical therapy since it is less invasive and often effective.
\end{abstract}

Keywords: gynecomastia, liposuction, subcutaneous mastectomy.

\section{Introduction}

Pesudogynecomastia or lipomastia, on the other hand, is a clinical disorder that occurs in obese patients because of an excess accumulation of fatty tissue without the growth of glandular tissue. The condition's prevalence has been shown to range anywhere from $4 \%$ to $70 \%$ in various studies. The large range of results is due to the fact that diverse methods of evaluation are being used. Aesthetic and psychological issues are prominent in patients with GM.

Dissociation and lack of self-confidence are common concerns for them. Because of worry and dread of breast cancer, many patients seek medical counsel on a frequent basis [2]. For moderate instances, reassurance, a healthy diet, and regular exercise are all that is needed for treatment. However, the most serious instances need a combination of pharmacological drugs and surgery. [3] Pathophysiology: Breast tissue contains oestrogen and androgen receptors. In contrast to androgen, which inhibits breast ductless growth, oestrogen promotes it. Genetically modified organisms (GM) may arise as a consequence of an imbalance between these two hormones. Even though there are several medical problems and drugs that have been linked to the development of GM, more than half of the cases are idiopathic. As Pituitary and adrenocortical cancers, for example, may be associated with the GM. In addition to HCG-producing tumours (carcinoma of lung, liver, and kidney, and choriocacarcinoma), there are other cancers that secrete excessive amounts of estradiol, such as sertoli or leydig cell tumours. Type 1 diabetes, pseudohermaphroditism, and hyperthyroidism are all examples of conditions when GM are present. Bodybuilders who take anabolic steroids to bulk up their muscles are more likely to suffer from Kennedy's condition, which GM has been proven to have. Druginduced GM accounts for around a quarter of all GM cases. Cancer chemotherapy, Spironolactone, Metoclopramide, Verapamil, Human Growth Hormone (HGH), Proton Pump Inhibitors (PPIs), and Highly Active Retroviral Therapy (HAART) are some of the medications that have been linked to GM. As a result of increased adiposetissue in the breast and higher activity of the aromatase enzyme conversion of testosterone to
E2 and rostendion to E1, as well as increased plasma estradiol concentrations while decreasing plasma testosterone concentrations, GM was seen in obese persons. [9]

\section{Subjects and Methods}

The study had been carried out at the department of General Surgery in faculty of medicine- Benha University. This study was carried out on 40 adolescent male patients aged between 18-35 yrs with gynecomastia ranging from grade I to early grade III.

Inclusion criteria: Age between 18-35yrs and grade I to early grade III

Exclusion criteria: Age above 35 or below 18 yrs, cases with co morbidity as; diabetes mellitus, CRF, liver cirrhosis, cardiac and hypertensive patients, late grade III or grade IV, patients under hormonal therapy, athletics with supplementation intake and psychologically unstable patients

\section{Methods}

All patients included in the study were subjected to the following:

Detailed history taking including: Personal data: Name, age, sex, occupation, address, a designed sheet was fulfilled for every patient to document his data, past history of previous interventions, hospital diagnosis, date of admission in hospital, medical \& Past history, hemodynamics (HR \& NIBP) and respiratory profile (RR \& SpO2)

Careful clinical examination: General: Vital signs (Blood pressure, Temperature, Heart rate, Respiratory rate) and signs of (Pallor, Cyanosis, Jaundice, and Lymph node enlargement).

Types of interventions: Subcutaneous liposuction with glandular excision through incision of lipo-suction.

Surgical technique: The surgical procedure is always performed under general anesthesia. Markings are done with the patient in an upright position and include: Inframammary fold, boundaries of liposuction and skin incisions, $8-12 \mathrm{~mm}$ long and set one behind the anterior axillary pillar and the other at the lateral extremity of the inframammary fold, due to the need for crossing liposuction tunnels and reaching the glandular tissue in the periareolar area. A hypotonic solution and 
local anesthetic with epinephrine 1:400,000 is used for subcutaneous infiltration. This step is now performed in a different way than before: the solution is injected through the two skin incisions using a 2-mm multi-hole smooth cannula. Twenty minutes after the solution has been injected, liposuction is performed. This is a wide and accurate liposuction with different aims: Fat tissue removal, breast contour remodeling and glandular tissue isolation. Fat tissue removal and contour reshaping are performed routinely in liposuction of the pectoral region. Isolation of the glandular tissue, however, is a specific step of this technique. Treating the superficial plane, we used a thinner cannula $(2-3 \mathrm{~mm})$ to separate the parenchyma from the subcutaneous tissue, freeing the superficial layer skin attachments. The two layers must be meticulously created keeping in mind that the goal of the procedure is to detach glandular tissue almost completely both from the inferior plane and the superficial one, once the parenchyma has been freed from its lower and superficial attachments. All the pieces of removed mammary tissue are collected and sent, as separated specimens from the right and left breast, for histological examination. Additional liposuction, using a thin cannula can eventually be performed to smooth out contour irregularities, especially at the margins of the pectoral area and along the anterior axillary pillar. A suction drain is inserted on each side and maintained for 24-48 h. Skin incisions are sutured in layers, and stitches are removed on the eighth postoperative day. A compressive dressing is applied at the end of surgery and removed at the time of stitches' removal; after that, an elastic garment has to be worn until the end of the first postoperative month.

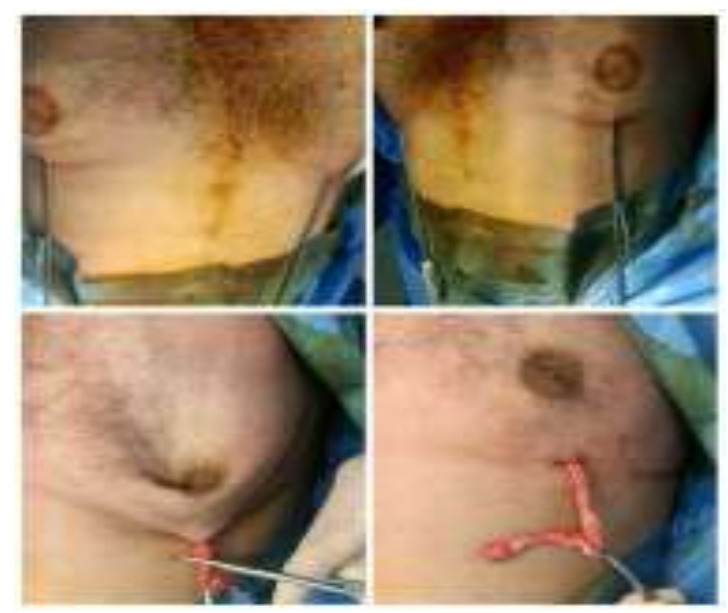

Fig. (1) Demonstrate our technique of inframammary liposuction then glandular excision. The same steps are employed on the other breast. Patients with prominent glandular tissue are in need for glandular excision which is excised through the same epsilateral stab after wound extension for $2 \mathrm{mms}$ extra length. Half-buried vertical mattress sutures are used for wound closure. Pressure garment is dressed from the immediate post-operative period. Patients are allowed to go home on the same day of surgery. Dressings are changed on the second post-operative day at outpatient clinics. We followed our patients at regular intervals at 1,3 , and 6 months post-surgery.

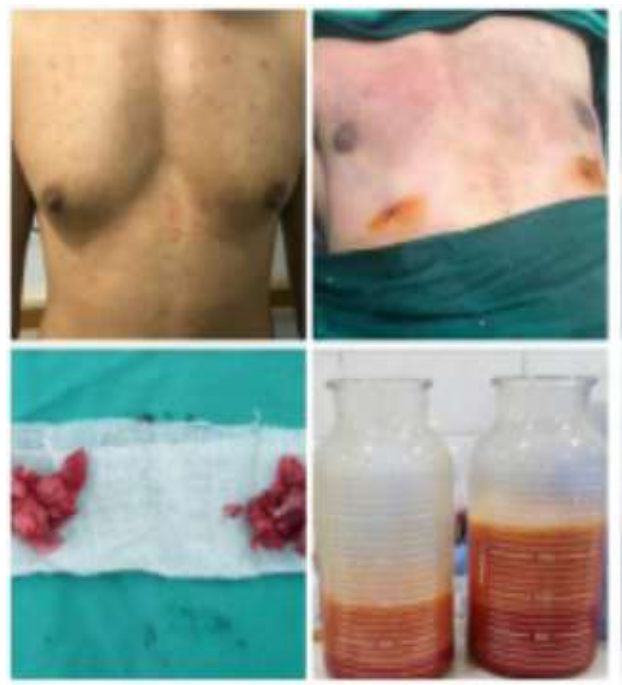

Fig. (2) Shows amount of liposuction, size of extracted gland, and post-operative result 
Administrative considerations: An Official permission was obtained from the ethical committee of the department of General Surgery, Faculty of Medicine, Benha University. An official permission was obtained from the Institutional Research. Approval from ethical committee in the faculty of medicine (Institutional Research Board IRB)

Ethical consideration: Informed consent was obtained from all participants after being informed about the aims and process of the study as well as applicable objectives. The study procedures were free from any harmful effects on the participants as well as the service provided.

Data management and Statistical Analysis: Data entry, processing and statistical analysis was carried out using using SPSS version 20(Statistical Package for the Social Sciences). Tests of significance (Kruskal-Wallis, Wilcoxon's, Chi square, logistic regression analysis, and Spearman's correlation) were used. Data were presented and suitable analysis was done according to the type of data (parametric and non-parametric) obtained for each variable. P-values less than $0.05(5 \%)$ was considered to be statistically significant.

\section{Results}

The mean age among grade $I$ is $25.5 \pm 3.9$. Also, the mean weight among grade III is $85.2 \pm 10.2$ and the

mean height among grade $\Pi$ is $170.3 \pm 20.8$. We found that mean BMI among grade III is 29.6 5.7. Table (1)

According to table 2 there is $50 \%$ of the participants have gynecomastia in right side and $45 \%$ had small gynecomastia. Only $20 \%$ of the participants have firm gynecomastia and 55\% had fair skin quality. Table (2)

There is $40 \%$ of the participants who described their main complain as lump only. Table (3)

Represent score satisfaction by three doctors not included in our study for evaluation the cases. The Likert scale for scar was among grade $I$ II $8.3 \pm 1.5$. Also, the Likert scale for contour among grade $\mathrm{I}$ is $6.5 \pm 2.4$. The mean score of complications is least among grade III $(2.9 \pm 2.1)$. The Likert scale for sensation among grade III is $8.1 \pm 1$. Table (4)

Regarding table 6, 45\% had ecchymosis, 35\% had pain, 20\% had skin irregularities and $15 \%$ had nipple necrosis after liposuction. Table (5)

Table (1) Basic characteristics of the participants $(\mathrm{N}=40)$

\begin{tabular}{lccc}
\hline Variables & Grade I & Grade II & Grade III \\
& Mean \pm SD & Mean \pm SD & Mean \pm SD \\
\hline Age (years) & $25.5 \pm 3.9$ & $26.4 \pm 5.6$ & $29.1 \pm 3.7$ \\
Weight (Kg) & $82.4 \pm 8.6$ & $83.7 \pm 9.7$ & $85.2 \pm 10.2$ \\
Height $(\mathbf{c m})$ & $169.8 \pm 15.3$ & $170.3 \pm 20.8$ & $173.5 \pm 21.5$ \\
BMI $(\mathbf{K g} / \mathbf{m} 2)$ & $25.7 \pm 4.2$ & $28.1 \pm 6.4$ & $29.6 \pm 5.7$ \\
\hline
\end{tabular}

Data are described as mean; SD, standard deviation

Table (2) Characteristics of breasts among the participants $(\mathrm{N}=40)$

\begin{tabular}{lc}
\hline Variables & $\mathbf{n}(\%)$ \\
\hline Side & $20(50)$ \\
Right & $14(35)$ \\
Left & $6(15)$ \\
Bilateral & \\
Size & $18(45)$ \\
Small & $12(30)$ \\
Medium & $10(25)$ \\
Large & \\
Consistency & $16(40)$ \\
Soft & $16(40)$ \\
Moderate & $8(20)$ \\
Firm & \\
Skin quality & $22(55)$ \\
Fair & $12(30)$ \\
Good & $6(15)$ \\
Striae & \\
\hline
\end{tabular}

Qualitative data are represented as frequencies and percentages 
Table (3) Main complain of the participants (N=40)

\begin{tabular}{lc} 
Complain & $\mathbf{n}(\%)$ \\
\hline Lump & $16(40)$ \\
Lump+ pain & $6(15)$ \\
Enlargement & $8(20)$ \\
Enlargement+ pain & $10(25)$ \\
\hline
\end{tabular}

Qualitative data represented as frequencies and percentages

Table (4) Likert scale among grades after intervention

\begin{tabular}{lccc}
\hline Variables & $\begin{array}{c}\text { Grade I } \\
\text { Mean } \pm \text { SD }\end{array}$ & $\begin{array}{c}\text { Grade II } \\
\text { Mean } \pm \text { SD }\end{array}$ & $\begin{array}{c}\text { Grade III } \\
\text { Mean } \pm \text { SD }\end{array}$ \\
\hline Scar & $7.6 \pm 1.9$ & $8.3 \pm 1.5$ & $8.1 \pm 1.5$ \\
Contour & $6.5 \pm 2.4$ & $7.1 \pm 3.1$ & $6.8 \pm 2.9$ \\
Complications & $3.2 \pm 2.5$ & $4.6 \pm 3.4$ & $2.9 \pm 2.1$ \\
Sensation & $6.9 \pm 3.2$ & $7.2 \pm 2.6$ & $8.1 \pm 1.8$ \\
\hline
\end{tabular}

Scores from 1-10, 1 least favorable and 10 the most favorable

Table (5) Complications after intervention among participants $(\mathrm{N}=40)$

\begin{tabular}{lc}
\hline Complications & $\mathbf{n}(\%)$ \\
\hline Partial nipple necrosis & $6(15)$ \\
NAC loss of sensation & $0(0)$ \\
Hematoma & $4(10)$ \\
Seroma & $6(15)$ \\
Pain & $14(35)$ \\
Skin irregularities & $8(20)$ \\
Ecchymosis & $18(45)$ \\
Residual skin /gland & $2(5)$ \\
Wound infection /dehiscence & $2(5)$ \\
\hline
\end{tabular}

Qualitative data are represented as frequencies and percentages

\section{Discussion}

Gynecomastia is a noncancerous enlargement of the male breasts caused by an overgrowth of glandular tissue, and it appears as an elastic or firm mass that grows outward from the nipple in a circular pattern. An estimated 40-65 percent of men suffer with this benign disease of the male breast. Surgeons who favour liposuction to remove glandular tissue say it is less invasive and leaves fewer scars than conventional subcutaneous mastectomy, but surgeons who support conventional subcutaneous mastectomy say that complete surgical excision of glandular tissue is required to treat gynecomastia. [10] For this research, the most important goal was to analyse the outcomes of the surgical procedure for gynecomastia therapy that is composed of infra-mammary liposuction and glandular excision.

This cross-sectional research comprised 40 teenage male patients with gynecomastia ranging from grade I to early grade III, who were between the ages of 18 and 35 . A follow-up of six months followed the research.

The average age of students in grade one is 25.5 3.9. Students in grades III have an average weight of 85.2 pounds, whereas those in grades II have an average height of 170.3 pounds, on average. The average BMI for students in grade III was 29.6 5.7.

Akhtar et al. [12] stated that 60 patients were separated into two groups in their research, which corroborated our findings. There were 30 patients in each group. An open disc excision was performed on Group A, while suction-assisted arthroscopic shaving disc excision was used on Group B. Patients ranged in age from 25.76 to 5.38 years on average. Twelve patients in Group B had disease of Grade II-A and eighteen in Group B had disease of Grade II-B.

Mean age of Rayamajhi et alpatients .'s was 53.24 15.266 Years, with the range of 26-69 years in their research [13]. $10(30.30 \%)$ had Simons grade I illness; $17(51.51 \%)$ had Simons grade IIA disease; and 6 (18.18\%) had Simons grade IIB disease.

Gynecomastia on the right side affects $50 \%$ of participants, while the remaining $45 \%$ suffer just minor symptoms. Most individuals had bilateral gynecomastia, while only $15 \%$ have just left gynecomastia and $35 \%$ have both. Among the study's subjects, only $20 \%$ had a firm gynecomastia and $55 \%$ had fair skin. Lumps were listed as the most common complaint by $40 \%$ of subjects.

However, in the research of Rayamajhi et al., [13], 19 of 32 patients had unilateral gynecomastia, while only 14 individuals had bilateral gynecomastia (59.37 percent). Only 12 of the unilateral instances included the right breast, while only 7 involved the left. 
601 patients got bilateral surgery and three underwent unilateral surgery, according to Kim et al. [14].

Aesthetic concerns about the GM's look were shown to be more common than physical malformation in a recent research by Couto et al. [15].

According to the present research, grade II liposuction patients were found to have an overall satisfaction score of 8.1-2.3. In addition, the grade I chest shape score is 6.52.4. Grade III students had the lowest average degree of worry about scars (2.9 2.1). Confidence among grade III students ranges from 8.1 to 1.8. Before and after liposuction, there is a statistically significant change in satisfaction ratings, chest shape, and self-confidence levels. After liposuction, the results are better.

Patient satisfaction with the operation was measured using the visual analogue scale in the research by Akhtar et al. [12] during follow-up visits. When comparing the open disc excision group to the arthroscopic shaver group, higher levels of satisfaction were found in the former $(8.6 / 10$ versus $7.8 / 10)$. There was a statistically significant difference in patient satisfaction between open disc excision and arthroscopic disc excision ( $\mathrm{p}=0.126)$. It is opposite to the findings of Paul et al., (16) who found that patients who had arthroscopic disc excision were more satisfied with the procedure.

The use of liposuction in the beginning helps to distinguish glandular tissue, making it easier to remove it and get a more aesthetically pleasing result. Better visibility of the tissue ensures full excision and greater control over bleeding using the open approach. There will be no scarring along the nipple from the suctionassisted arthroscopic nipple procedure since it utilises the same incision as the first liposuction procedure. Small bleeding spots might lead to an increased risk of postoperative hematoma development, therefore this does not ensure full excision. The continual oscillating action of the shaver might potentially lead to necrosis if it is not conducted correctly. [17]

Aboelatta et al. [18] found that patients in group I had a high to outstanding chance of survival. Group II patients, on the other hand, had similarly positive outcomes. 30 male patients with grades IIa and IIb were included; all were men. Group I included 15 patients who had laser-assisted liposuction followed by endoscopic glandular tissue removal, while group II included 15 patients who had conventional liposuction with vacuum assistance followed by endoscopic glandular tissue excision (group II).

A total of 219 individuals (384 breasts) ranging in age from 12 to 74 years old were studied by Wong et al. [19]. In $24 \%$ of breasts, UAL was used (47 patients, 91 breasts). With conventional liposuction, UAL had much lower intraoperative and postoperative rates of open excision and revision (25 percent and 2 percent, respectively, compared to 39 percent and 19 percent, respectively, for conventional liposuction).
Researchers found that, on average, patients in $\mathrm{Oh}$ \& Lee's research [20] reported a satisfaction level of $4.13(0.5)$, with the vast majority of those who took part rating themselves as happy or very satisfied. Preoperative "dissatisfaction" on the BEQ (Breast Evaluation Questionnaire) climbed to "satisfaction" on the BEQ (Breast Evaluation Questionnaire) postoperatively from a "dissatisfied" score of $1.8(0.2)$. In the six months after surgery, the patients' mean (SD) overall satisfaction ratings with scars were $4.39(0.49)$, 4.65 (0.48), 4.78 (0.42), 4.82 (0.38), 4.78 (0.42), and $4.47(0.66)$

In a prior research, it was shown that bad chest contours before the procedure were improved to a score of 7.76 after the operation by three observers. Chest symmetry improved postoperatively from a satisfactory preoperative score of 5.94 to 8.73. Skin sagging had a score of 3.94 before to the procedure, but it rose to 7.94 after it. There was a high demand for first surgery (2.37), but little demand for revision surgery (8.76). Scarring after surgery was almost always a positive experience (9.01). In the case of gynecomastia treatment, the analysis of variance (ANOVA) showed a p-value of 0.002 and an F-statistic value of 29.352, indicating a substantial improvement in outcomes. (123)

Iwuagwu et al. [21], for example, found good cosmesis, decreased analgesia requirements, and high patient satisfaction rates in their investigations.

Setta et al. [22], based on their findings, reported that there were 20 participants; 10 in each group. The laser aided group had a median satisfaction score of 4.5, whereas the ultrasound group had a satisfaction score of 3.4. According to Couto et al., [15], all outcomes were rated as excellent or good by patients and surgeons alike.

Rayamajhi et al. [13], showed that the majority of patients (63.6 percent) replied as an exceptional patient satisfaction rating in the study's final results.

45 percent of patients experienced ecchymosis, 35 percent had discomfort, 20 percent had skin abnormalities, and 15 percent had necrosis of the nipple after liposuction, according to our findings. Only 5\% of the subjects experienced a wound infection/dehiscence, and only $5 \%$ had remaining skin/glands that were infected.

Only two patients with open disc excision and three patients with arthroscopic disc excision had hematoma development, according to Akhtar et al. [12]. Both groups of patients did not have necrosis of the nipple. There was no difference between the two groups when it came to the development of contour deviations. It was shown that eight individuals had asymmetry in the open disc excision, whereas 10 patients had similar issue in the arthroscopic disc excision. Both groups had the same level of tolerance for scarring. There were only two reoperations in arthroscopic disc excision.

There were no cases of nipple-areola complex displacement, necrosis, or retraction, post-procedure haemorrhage, infection, skin necrosis, or asymmetry in the research conducted by Couto et al., [15]. There were 
no reports of decreased nipple feeling or changes in erection in any of the patients. Bruises and hematomas developed in all patients, but they disappeared on their own within 30 days.

While in the Rayamajhi et al. [13] investigation, three seroma, one hematoma, and one wound dehiscence occurred as acute complications after the operation. In one example, a later mammogram revealed irregularities in the breasts. There were a total of six situations in which problems arose, but all were handled effectively. Two of the total number of surgeries were not followed up on.

Studying complications, researchers found one incidence of under-correction in group A and one case of dimpling deformity with under-correction in group B (Kim and colleagues, 2014) [14]. The patient in group A who was undercorrected got a second liposuction/subcutaneous mastectomy combo operation three years after the first. The patient gave himself a satisfaction score of 7 two years following the reoperation.

\section{Conclusion}

Gynecomastia is a benign abnormal enlargement of the male breast due to proliferation of glandular tissue, which presents as a rubbery or firm mass extending concentrically from the nipple. It is the most common benign condition of the male breast and is estimated to affect about 40-65 \% of males. Gynecomastia has several causes, including an imbalance in the testosterone-to-estrogen ratio in male breast tissue. The primary mechanisms include decreased androgen production, increased estrogen production, and increased availability of estrogen precursors for peripheral conversion to estrogen.

\section{References}

[1] FQ.Nuttall. Gynecomastia as a physical finding in normal men. J Clin Endocrinol Metab.vol. 48,pp. 338-340,1979.

[2] H.Rosen, ML.Webb, AD.DiVasta, AK.Greene, CB.Weldon, H.Kozakewich. Adolescent gynecomastia: not only an obesity issue. Ann PlastSurg.vol. 64,pp. 688-690,2021.

[3] P.Gikas, K.Mokbel. Management of gynaecomastia: anupdate. Int J ClinPract.vol. 61,pp.1209-1215, 2007.

[4] H.Rosen, ML.Webb, AD.DiVasta. Adolescent gynecomastia: not only an obesity issue. Ann Plast Surg.vol. 64(5),pp.688-90,2010.

[5] GD.Braunstein, N.Gynecomastia. Engl J Med.vol.328(7),pp.490-5,1993.

[6] G.Pearlman, HE.Carlson.Gynecomastia: an update. The Endocrinologist.vol.16(2),pp.109-15, 2006.

[7] G.Magro, P.Gangemi, L.Villari, P.Greco. Deciduoid-like stromal cells in a diabetic patient with bilateral gynecomastia: a potential diagnostic pitfall.Virchows Arch.vol.445(6),pp.659 60,2004.
[8] RD.Goldman. Drug-induced gynecomastia in children and adolescents. Can Fam Physician.vol. 56(4),pp.344-5,2010.

[9] S.Loves, J.Ruinemans-Koerts, H.de Boer. Letrozole once a week normalizes serum testosterone in obesity-related male hypogonadism. Eur J Endocrinol .vol. 158,pp. 741-747,2008.

[10] GA.Kanakis, L.Nordkap, AK.Bang, AE.Calogero, G.Bártfai, G.Corona. EAA clinical practice guidelines-gynecomastia evaluation and management. Andrology.vol. 7(6),pp.778-93, 2019.

[11] M.Tashkandi, MM. Al-Qattan, JM. Hassanain, MB.Hawary, M. Sultan. The surgical management of high-grade gynecomastia. Ann Plast Surg.vol. 53,pp.17-21,2004.

[12] A.Akhtar, F.Eitezaz, M.Rashid, I.Khan, SA.Malik. Liposuction in gynecomastia: an assessment of the suction-assisted arthroscopic shaver versus open disc excision techniques. Cureus.vol.8,pp. 11(10), 2019.

[13] J.Rayamajhi, S.Rawal, S.Malla. Liposuction in gynecomastia: an institutional experience. Medical Journal of Shree Birendra Hospital. Dec.vol. 23,pp.16(2):37-40, 2017.

[14]DH.Kim, IH.Byun, WJ.Lee, DK.Rah, JY.Kim, DW. Lee. Surgical management of gynecomastia: subcutaneous mastectomy and liposuction. Aesthetic plastic surgery. Dec.vol. 40(6),pp.87784, 2016.

[15] HL.Couto, CN.Valadares, OP.Junior, TC.Oliveira, PM.El Bacha, SD.Ferreira. Minimally invasive treatment of gynecomastia by ultrasound-guided vacuum-assisted excision: report of a case series. Mastology (Online).vol.8,pp.1-7, 2021.

[16]P.Petty, M.Solomon, E.Buchel, N.Tran. Gynecomastia: evolving paradigm of management and comparison of techniques. Plast Reconstr Surg.vol. 125,pp.1301-1308,2010.

[17] J.Benito-Ruiz, M.Raigosa, M.Manzano, L.Salvador. Assessment of a suction-assisted cartilage shaver plus liposuction for the treatment of gynecomastia. Aesthet Surg J.vol. 29,pp.302309, 2009.

[18] YA.Aboelatta, MM.Abdelaal. Comparison of laser-assisted liposuction and traditional liposuction combined with endoscopic surgical excision of grade II gynecomastia. European Journal of Plastic Surgery. Dec.vol.40(6),pp.5418, 2017.

[19] KY.Wong, CM. Malata. Conventional versus ultrasound-assisted liposuction in gynaecomastia surgery: a 13-year review. J Plast Reconstr Aesthet Surg.vol. 67(7),pp.921-6, 2014.

[20] YH.Oh, SH.Lee. Liposuction Using Wall Suction and Portable Suction in the Treatment of Gynecomastia. Indian Journal of Surgery. Mar.vol. 18,pp.1-7, 2021. 
[21] OC.Iwuagwu, TA.Calvey, D.Ilsley, PJ.Drew. Ultrasound guided minimally invasive breast surgery (UMIBS): A superior technique for gynaecomastia. Ann Plast Surg.vol.52,pp.131-3, 2004.
[22] HS.Setta, KA.Reyad, NF.AlMahmoudy. YAG Laser versus Ultrasound Assisted Liposuction in the Management of Grade II Gynecomastia (Comparative Study). The Egyptian Journal of Plastic and Reconstructive Surgery. Oct .vol.1;43(3),pp.469-74, 2020. 